\title{
Estratégia da Saúde da População Negra - Atenção Básica de Saúde
}

\author{
Black Population Health Strategy - Primary Health Care
}

Estrategia de salud de lapoblación negra - Atencion primaria de salud

Carlos Damião de Araujo Lima ${ }^{*}$, Margarida Maria Donato dos Santos²

Como citar esse artigo. Silva, AA; de La Longuiniere, ACF; Souza, IA; Cafezeiro, AS; Cunha, ALGO; Yarid, SS. A espiritualidade no processo de formação dos profissionais de saúde. Revista Pró-UniverSUS. 2020 Jul./Dez.; 11 (2): 141-144.

\author{
Resumo
}

Este estudo pretende analisar a assistência e cuidados dispensados a Saúde da População Negra, no contexto da Estratégia da Saúde da Família. Durante minha vivência na Atenção Básica, e voltado as questões étnicas de nosso País, com relação a saúde, observei que os tratamentos aos usuários na Atenção Básica, são iguais para brancos e negros, deveria haver um tratamento diferenciado aos usuários negros, em virtude de algumas patologias serem exclusivas da raça Negra. Algumas especificidades devem ser vistas nos cuidados da saúde desta população. Visando contribuir para um melhor tratamento na assistência pelos enfermeiros na Atenção Básica à população negra, realizamos este trabalho, é uma revisão bibliográfica, estudos com base em Ciências da Saúde LILACS e BVS/BIREME. Existem poucas pesquisas realizadas a respeito dessa temática, observa-se também que nas Instituições de Ensino Superior não temos estudos na Graduação de Enfermagem sobreeste assunto.

Palavras-chave: Atenção Básica, Enfermagem, Saúde População Negra.

Abstract
This study aims to analyze the care and care provided to Health of the Black Population, in the context of the Family Health
Strategy. During my experience in Primary Care, and addressing the ethnic issues of our country, regarding health, I observed
that treatments for users in Primary Care are the same for whites and blacks, there should be a differentiated treatment for
black users, because of some pathologies are unique to the Black race. Some specifics should be seen in the health care of
this population. Aiming to contribute to a better treatment in the assistance by nurses in Primary Care to the black population,
we conducted this work, is a literature review, studies based on Health Sciences LILACS and BVS / BIREME. There are few
studies on this subject, it is also observed that in the Higher Education Institutions we have no studies in Nursing Undergraduate
on this subject.

Keywords: Primary Care, Nursing, Health Black Population.

\section{Resumen}

Este estudio tiene como objetivo analizar la atención y la atención prestada a Salud de la población negra, en el contexto de la Estrategia de salud familiar. Durante mi experiencia en Atención Primaria, y al abordar los problemas étnicos de nuestro país, con respecto a la salud, observé que los tratamientos para los usuarios de Atención Primaria son los mismos para los blancos y los negros, debido a que debería haber un tratamiento diferenciado para los usuarios negros, debido Algunas patologías son exclusivas de la raza negra. Se deben ver algunos detalles en la atención médica de esta población. Con el objetivo de contribuir a un mejor tratamiento en la asistencia de enfermeras en Atención Primaria a la población negra, realizamos este trabajo, es una revisión de la literatura, estudios basados en Ciencias de la Salud LILACS y BVS / BIREME. Existen pocos estudios sobre este tema, también se observa que en las Instituciones de Educación Superior no tenemos estudios de Pregrado en Enfermería sobre este tema.

Palabras clave: Atención primaria, Enfermería, Salud Población Negra.

\section{Introdução}

A Política Nacional da Saúde Integral da População Negra abrange ações e programas de diversas secretarias e órgãos vinculados ao Ministério da Saúde (MS). Trata-se portanto, de uma política transversal, com formulação, gestão e operação compartilhadas entre as três esferas de governo, de acordo com os princípios e diretrizes do SUS ${ }^{1}$.

A Referida Política define os princípios, a marca, os objetivos, as diretrizes, as estratégias e as responsabilidades de gestão, voltados para a melhoria das condições de saúde desse segmento da população, inclui ações de cuidado, atenção, promoção à saúde e prevenção de doenças, bem como de gestão participativa, controle social, produção de conhecimento, formação 
e educação permanente para trabalhadores de saúde, participação popular, visando à promoção de equidade em saúde da população negra ${ }^{2: 15}$.

Na década de 1990 o governo federal passou a se ocupar do tema, em atenção as reivindicações da Marcha Zumbi dos Palmares, realizada em 20 de Novembro de $1995^{1}$. O que resultou na criação do Grupo de Trabalho Interministerial para a Valorização da população Negra/ GTI e do Subgrupo Saúde. Em Abril do ano seguinte, o GTI organizou a Mesa-Redonda sobre Saúde da População Negra, cujos principais resultados foram: a introdução do quesito cor nos sistemas de informação de mortalidade e de nascidos vivos; na elaboração da Resolução CNS 196/96, que disciplina aspectos éticos das pesquisas em seres humanos, foi introduzido o recorte racial em toda e qualquer pesquisa; a recomendação da formação de uma política nacional de atenção às pessoas com anemia falciforme ${ }^{3,2}$.

O presente estudo tem por objeto de estudo o cuidado, o tratamento e o acolhimento da Enfermagem aos usuários negros na Atenção Básica de Saúde, face as diversas patologias exclusivas para essa importante parte da sociedade brasileira. Assim, objetivamos analisar a assistência e cuidados dispensados a Saúde da População Negra, no contexto da Estratégia da Saúde da Família.

\section{Metodologia}

Trata-se de uma pesquisa feita através de levantamento bibliográfico, com coleta de dados a partir de fontes secundárias ${ }^{4}$. A pesquisa bibliográfica tem o objetivo de proporcionar maior familiaridade com o problema, com vistas a torná-lo mais explícito. A coletânea de informações hoje existentes na Internet é um grande passo para os pesquisadores, tornando o acesso, fácil para todos que querem se informar e se atualizar frequentemente.

A revisão literária busca compilar conhecimentos sobre os tópicos e ajudando nas bases de um importante estudo para a enfermagem. Essa responsabilidade é capital para os pesquisadores.

Foram utilizados documentos escritos (artigos científicos) referentes ao assunto, publicados entre 2014 e 2018 onde se constatou o maior numero de produções cientificas relacionada ao tema em questão, selecionouse a partir dos seguintes critérios de inclusão: ser artigo escrito por pelo menos um profissional enfermeiro; estar publicado em um dos periódicos encontrados para o estudo; estar o artigo disponível na íntegra no banco de dados online e apresentar os descritores e suas combinações: Atenção Básica, Grupos com ancestrais do Continente Africano, Enfermagem.

Para o levantamento dos artigos de literatura, realizou-se uma busca nas seguintes bases de dados: Literatura Latino-Americana e do Caribe em
Ciências da Saúde (LILACS), Medical Literatura AnalysisandRetrievalSistem on-line (Medline), Google Acadêmico, Pubmed, e BVS/BIREME.

O período de coleta dos dados foi de Agosto a Setembro de 2019 e posteriormente foram analisados criteriosamente. $\mathrm{O}$ material coletado foi analisado a partir de uma planilha, onde os dados foram agrupados de acordo com pontos de convergência, reduzidos para realizar o processo de codificação e serão discutidas as categorias do estudo.

Os critérios de inclusão definidos para a seleção dos artigos foram: artigos publicados em português, inglês e espanhol; artigos na íntegra que retratassem a temática referente à Saúde da População Negra e artigos publicados e indexados nos referidos bancos de dados nos últimos dez anos.

\section{Resultados}

A amostra final desta revisão foi constituída por cinco artigos científicos selecionados pelos critérios de inclusão previamente estabelecidos, publicados na base de dados da BVS/BIREME no período de 2014 a 2018, que abordavam os descritores selecionados.

Após a leitura do material, estabelecemos uma caracterização destes artigos quanto ao local, ano e método de pesquisa realizada. Desta forma, podese perceber a incipiência de artigos científicos sobre o assunto, uma vez que se trata de uma metodologia baseada na Prática Baseada em Evidências (PBE), que se encontra em franco desenvolvimento, não apenas na Enfermagem, mas em todas as disciplinas da Área da Saúde.

Nota-se uma expressiva falta de discussões sobre a Enfermagem na Saúde da População Negra, apesar da PNISPN, esperamos que breve seja realmente implantada essa Política para um atendimento de qualidade a essa importante parte da sociedade brasileira.

Os profissionais de enfermagem que atuam na Atenção Básica de Saúde, têm ainda, além de pouco conhecimento das patologias que afetam aos negros, um outro grave problema, que é a forma de abordagem ao usuário, em virtude do preconceito existente: como dizer ou explicar ao usuário negro que o tratamento para ele é diferente do usuário branco, quando os dois possuem a mesma patologia, por exemplo a HAS. Os enfermeiros deveriam passar por cursos para conhecimento específicos dessas patologias para melhor esclarecimento a esses usuários.

Após a leitura do material, estabelecemos uma caracterização destes artigos quanto aos temas das pesquisas realizadas.

$\mathrm{Na}$ área de saúde, o racismo institucional é presente à medida que o atendimento pauta-se na desigualdade ao atenderem a população a partir de um olhar racial. Sendo de fundamental importância uma 
discussão no decorrer do processo formativo em saúde, para compreensão de se pensar políticas públicas. ${ }^{5}$

Barreiras de acessibilidade se interpõem entre a oferta de serviços e o atendimento efetivo da população negra. $\mathrm{O}$ acesso é permeado pela demora no atendimento, falta de compromisso dos profissionais de saúde. A discriminação nos serviços de saúde, devem fazer parte das discussões já no processo formativo na área da saúde, trazendo para a discussões as questões materiais e simbólicas, sendo indispensável para se pensar em equidade no SUS. ${ }^{6}$

Medidas educativas constituem-se importantes estratégias de prevenção que visam à melhoria da saúde e de qualidade de vida, além de contribuir com a saúde da população negra. Contudo para que esse processo ocorra, torna-se essencial a promoção se ações que tragam em seu bojo, informações que considerem as necessidades da população assistida, sobretudo o entendimento dessa população sobre os fatores de risco.

Enquanto atividade sistematicamente planejada e inerente ao processo de cuidar, a educação em saúde, facilita, predispõe e reforça medidas comportamentais adotadas por uma pessoa, grupo ou comunidade para alcançar um efeito intencional sobre a própria saúde. ${ }^{7}$

A luta por melhores condições para a população negra ainda é restrita, atendo suas demandas em saúde ao cuidado de pessoas com doença falciforme e em alertas sobre a maior prevalência de miomas uterinos entre as mulheres negras. A Política Nacional de Saúde integral da População Negra, aborda condições da atenção,

Quadro 1. Apresentação dos dados dos artigos que compuseram a amostra.

\begin{tabular}{|c|c|c|c|c|}
\hline Procedência & $\begin{array}{l}\text { Título do } \\
\text { Artigo }\end{array}$ & Autores & Publicação & Temática \\
\hline BVS/Bireme & $\begin{array}{l}\text { O Conceito de } \\
\text { racismo } \\
\text { institucional, } \\
\text { aplicações no } \\
\text { campo da } \\
\text { Saúde }^{5}\end{array}$ & $\begin{array}{l}\text { Laura Cecilia } \\
\text { Lopez }\end{array}$ & $\begin{array}{l}\text { Interface } \\
\text { (Botocatu) } \\
\text { Vol16no.40. } \\
\text { Botucatu } \\
\text { Jan/mar.2012- } \\
\text { Epub.Mar20,2012 }\end{array}$ & $\begin{array}{l}\text { Conceito Racismo } \\
\text { Institucional. } \\
\text { Promoção da } \\
\begin{array}{lr}\text { da Paúde } \\
\text { redução }\end{array} \\
\begin{array}{l}\text { desigualdades } \\
\text { prioridade }\end{array} \\
\end{array}$ \\
\hline BVS/Bireme & $\begin{array}{l}\text { Acessibilidade } \\
\text { a atenção } \\
\text { básica a } \\
\text { familias negras } \\
\text { em bairro } \\
\text { pobre } \quad \mathrm{em} \\
\text { Salvador- } \\
\text { Brasil }^{6}\end{array}$ & $\begin{array}{l}\text { Leny Alves } \\
\text { Bomfim, } \\
\text { et.al }\end{array}$ & $\begin{array}{l}\text { Revista da Saúde } \\
\text { Pública,2012;46(6) } \\
; 1007-13\end{array}$ & $\begin{array}{l}\text { Analisar } \\
\text { acessibilidade de } \\
\text { familias negras em } \\
\text { bairro popular aos } \\
\text { serviços básicos de } \\
\text { saúde de Salvador- } \\
\text { Brasil }\end{array}$ \\
\hline BVS/Bireme & $\begin{array}{l}\text { Hipertensão } \\
\text { arterial- } \\
\text { Conhecimento } \\
\text { de Jovens } \\
\text { quilombolas? }\end{array}$ & $\begin{array}{l}\text { Thais de } \\
\text { Andrade } \\
\text { Alves, et.al }\end{array}$ & $\begin{array}{l}\text { Revista Brasileira } \\
\text { de } \\
\text { Funcional- } \\
\text { Cachocira-Bahia- } \\
\text { V.I, N.II.P. } \\
\text { Jun.2016 }\end{array}$ & $\begin{array}{l}\text { Identificar os saberes } \\
\text { dos adolescentes } \\
\text { jovens quilombolas a } \\
\text { cercu da HAS }\end{array}$ \\
\hline BVS/Bireme & $\begin{array}{l}\text { Promoçào da } \\
\text { Equidade em } \\
\text { Saúde para a } \\
\text { Populaçào } \\
\text { Negra }^{8}\end{array}$ & $\begin{array}{ll}\text { Ana Maria } \\
\text { Costa }\end{array}$ & $\begin{array}{l}\text { BIS, Bol. lnst. } \\
\text { Saúde } \\
\text { (Impr.) vol.13 no.2 } \\
\text { São } \\
\text { Paulo out. } 2011\end{array}$ & $\begin{array}{l}\text { Discute a necessidade } \\
\text { do SUS, formular e } \\
\text { implementar Politicas } \\
\text { de saúde para a } \\
\text { populaçào Negra }\end{array}$ \\
\hline BVS/Bireme & $\begin{array}{l}\text { Iniquidades } \\
\text { raciais e saúde: } \\
\begin{array}{ll}0 \quad \text { ciclo da } \\
\text { politica de } \\
\text { saúde da } \\
\text { população } \\
\text { negra }^{9}\end{array}\end{array}$ & $\begin{array}{l}\text { Luís Eduardo } \\
\text { Batista I; } \\
\text { Rosana } \\
\text { Batista } \\
\text { Monteiro II; } \\
\text { Rogério } \\
\text { Araujo } \\
\text { Medeiros III }\end{array}$ & $\begin{array}{l}\text { Saúde em Debate, } \\
2013,37: 681-690\end{array}$ & $\begin{array}{l}\text { Discute o ciclo da } \\
\text { Política de Saúde da } \\
\text { População Negra }\end{array}$ \\
\hline
\end{tabular}


incluindo o racismo como determinante social da saúde. Compondo esse cenário, debates sobre impasses, lacunas, desafios e as dificuldades na implementação e avanços conquistados, constitui-se passo fundamental para se pensar políticas públicas. Visando a consolidação da universalidade do acesso à saúde, com a redução das desigualdades, assumindo contudo, o racismo como um determinante de desigualdade social diretamente relacionada à condição de saúde. ${ }^{8}$

Fator de violação de direitos e de produção de iniquidades, especialmente no campo da saúde, e no âmbito da saúde, o racismo com fenômeno ideológico está presente na qualidade do cuidado e assistência, em sofrimentos evitáveis, em taxas de mortalidade da população negra. Para tanto, iniciativas, ações e políticas, tem subsidiado a implementação de novas tecnologias, desenvolvidas para se discutir e trabalhar temas relacionados às iniquidades raciais com profissionais de saúde. Utilizando a educação permanente como o intuito de formação e capacitação profissional. ${ }^{9}$

\section{Discussão}

Percebe-se a incipiência de artigos científicos sobre o assunto, uma vez que se trata de uma metodologia baseada na Prática Baseada em Evidências (PBE), que se encontra em franco desenvolvimento, não apenas na Enfermagem, mas em todas as disciplinas da Área da Saúde.

No contexto dos autores, nota-se uma preocupação com a saúde da população negra, tanto no cuidado específico de uma patologia (HAS) por exemplo, como também para que essa população tenha acesso ao conhecimento de suas doenças e seus direitos. A Educação Permanente é aplicada e desenvolvida em cursos específicos sobre diversas patologias com conteúdo estratégicos e objetivos. Com foco nos profissionais da saúde o curso contou com significativa presença de profissionais da educação pública municipal e estadual, das cidades de São Luis e Pinheiro, no Maranhão, implicando a discussão do tema " Saúde da População Negra (especialmente da mulher negra).

A PNISPN ainda se encontra em processo de implantação, dependendo da área, educação, saúde, direito e segurança dentre outros. Nota-se que o acesso e acessibilidade atraem cada vez mais atenção dos pesquisadores da saúde coletiva. Mas esbarramos em muitas dificuldades nos municípios mais afastados dos grandes centros urbanos, é quase nula o acesso desta população à saúde, seja porque as Unidades Básicas não recebem recursos necessários ou por falta de interesse dos usuários, quando vão a demora é bastante longa para um atendimento médico, ou a marcação de um exame, preferindo os antigos e tradicionais tratamentos caseiros, passados seus avós.

\section{Considerações Finais}

Este estudo mostra que o atendimento dos enfermeiros da Atenção Básica de Saúde à População Negra, face as suas patologias específicas, não se diferenciam da pessoa branca, as consultas de enfermagem, são feitas de forma igual para brancos e negros, inclusive o pré-natal da mulher negra.

Com isto constata-se a falta de orientações a esses profissionais quanto a saúde desta população que por suas particularidades devem ser atendidos de forma diferenciada. Evidencia-se a necessidade de pesquisas sobre a temática, uma vez que são poucas as publicações periódicas envolvendo o cuidado da saúde da população na Atenção Básica.

Aliado a isto, torna-se necessário rever a formação dos profissionais de saúde para que no processo de aprendizado sejam discutidas formas de cuidado específico á saúde dessa população, desde a anatomia até a terapêutica, pois o ensino é feito a partir das pesquisas em corpos brancos o que dificulta o aprendizado do aluno para situações específicas como identificar manchas nos corpos negros, preparando desta forma o futuro profissional para lidar com as diretrizes da política de saúde que direciona o cuidado à saúde da população negra.

É preciso que seja mais utilizado a integralidade na assistência à saúde da população negra, uma das diretrizes do SUS, que considera a saúde sob uma perspectiva ampla e complexa abrangendo ciclos de vida, riscos e vulnerabilidades, níveis de complexidade de assistência. Além de equidade e humanização dos serviços, pois assim estaremos caminhando para uma assistência mais qualificada e direcionada a saúde da população negra, caracterizando o princípio da igualdade, tão difundido na política do Sistema Único de Saúde - SUS.

\section{Referências}

1.Brasil. Ministério da Saúde, (2008). Política Nacional de Saúde Integral da População Negra - Plano Operativo. Disponível em: bvsms.saude.gov.br/.../ politica_populacao_negra_plano_operativo.p.

2.Inocêncio JF, Tavares MM. A percepção do enfermeiro quanto à saúde do negro. Revista Pró-UniverSUS, 2014;5(2), 11-17.

3.PNUD, OPAS. Política nacional de saúde da população negra: uma questão de equidade. Brasília: Programa das Nações Unidas para o Desenvolvimento/ PNUD e da Organização Pan-Americana da Saúde/OPAS. 2001. Workshop Interagencial de Saúde da População Negra. Disponível em: http://www. unaids.org.br/biblioteca/links/PNUD/PNUD\%202.pdf.

4.Gil AC. Métodos e técnicas de pesquisa social. São Paulo: Atlas, 2002.

5.Lopez LC. O Conceito de racismo institucional, aplicações no campo da SaúdeInterface. 2012, Jan/mar:;16(40).

6.Bomfim LA, et.al. Acessibilidade a atenção básica a famílias negras em bairro pobre em Salvador-Brasil. Revista da Saúde Pública,2012;46(6);100713.

7.Alves TA, et.al. Hipertensão arterial-Conhecimento de Jovens quilombolas. Revista Brasileira de Saúde Funcional-Cachoeira-Bahia- 2016, Jun.1(2):3949.

8.Costa AM. Promoção da Equidade em Saúde para a População Negra. BIS Bol Inst. Saúde (Impr.) São Paulo out. 2011.;1.13(2)

9.Batista LEO, Monteiro RB, Medeiros RA. Iniquidades raciais e saúde: o ciclo da política de saúde da população negra. 2013, 37:681-690. 